\title{
Fluorescence-Guided Visualization of Soft-Tissue Sarcomas by Targeting Vascular Endothelial Growth Factor A: A Phase 1 Single-Center Clinical Trial
}

\author{
Pieter J. Steinkamp*1, Bobby K. Pranger*1, Mei-Fang Li ${ }^{2}$, Matthijs D. Linssen ${ }^{3,4}$, Floris J. Voskuil ${ }^{5}$, Lukas B. Been ${ }^{1}$, \\ Barbara L. van Leeuwen ${ }^{1}$, Albert J.H. Suurmeijer ${ }^{6}$, Wouter B. Nagengast ${ }^{4}$, Schelto Kruijff ${ }^{1,7}$, Robert J. van Ginkel ${ }^{1}$, \\ and Gooitzen M. van Dam ${ }^{1,7,8}$ \\ ${ }^{1}$ Department of Surgery, University Medical Center Groningen, University of Groningen, Groningen, The Netherlands; ${ }^{2}$ ChangJiang \\ Scholar's Laboratory, Shantou University Medical College, Shantou, China; ${ }^{3}$ Department of Gastroenterology and Hepatology, \\ University Medical Center Groningen, University of Groningen, Groningen, The Netherlands; ${ }^{4}$ Department of Clinical Pharmacy and \\ Pharmacology, University Medical Center Groningen, University of Groningen, Groningen, The Netherlands; ${ }^{5}$ Department of Oral \\ and Maxillofacial Surgery, University Medical Center Groningen, University of Groningen, Groningen, The Netherlands; \\ ${ }^{6}$ Department of Pathology and Medical Biology, University Medical Center Groningen, University of Groningen, Groningen, The \\ Netherlands; ${ }^{7}$ Medical Imaging Center, Department of Nuclear Medicine and Molecular Imaging, University Medical Center \\ Groningen, University of Groningen, Groningen, The Netherlands; and ${ }^{8}$ AxelaRx/TRACER BV, Groningen, The Netherlands
}

Resection of soft-tissue sarcoma (STS) is accompanied by a high rate of tumor-positive surgical margins (14\%-34\%), which potentially lead to decreased disease-free survival. Vascular endothelial growth factor $A$ is overexpressed in malignant tumors, including STS, and can be targeted with bevacizumab-800CW during fluorescence-guided surgery for real-time tumor detection. In this phase 1 clinical trial, we determined the feasibility, safety, and optimal dose of bevacizumab-800CW for fluorescence-guided surgery in STS for in vivo and ex vivo tumor detection. Methods: Patients with a histopathologic diagnosis of STS were included. In the dose-escalation phase, patients received bevacizumab-800CW intravenously $3 \mathrm{~d}$ before surgery $(10,25$, and $50 \mathrm{mg} ; n=8)$. In the subsequent doseexpansion phase, 7 additional patients received bevacizumab$800 \mathrm{CW}$ at the optimal dose. Fluorescence images were obtained in vivo and ex vivo during all stages of standard care. The optimal dose was determined by calculating in vivo and ex vivo tumor-tobackground ratios (TBR) and correlating these results with histopathology. Results: Fifteen patients with STS completed this study. All tumors could be visualized during in vivo and ex vivo imaging. The optimal bevacizumab- $800 \mathrm{CW}$ dose proved to be $10 \mathrm{mg}$, with a median in vivo TBR of $2.0( \pm 0.58)$ and a median ex vivo TBR of 2.67 ( \pm 1.6$)$. All 7 tumor-positive margins could be observed in real time after surgical resection. Conclusion: GS using $10 \mathrm{mg}$ of bevacizumab- $800 \mathrm{CW}$ is feasible and safe for intraoperative imaging of STS, potentially allowing tumor detection and margin assessment during surgery. An additional follow-up phase 2 study is needed to confirm the diagnostic accuracy.

Received Mar. 23, 2020; revision accepted Jun. 23, 2020

For correspondence or reprints contact: Gooitzen M. van Dam, University Medical Center Groningen, P.O. Box 30.001, 9700 RB Groningen, The Netherlands.

E-mail: g.m.van.dam@umcg.n

*Contributed equally to this work.

Published online Jul. 17, 2020.

COPYRIGHT (c) 2021 by the Society of Nuclear Medicine and Molecular Imaging.
Key Words: soft-tissue sarcoma; fluorescence-guided surgery; molecular imaging; vascular endothelial growth factor $A$; tumor targeting; near-infrared fluorescence

J Nucl Med 2021; 62:342-347

DOI: 10.2967/jnumed.120.245696

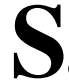
cers diagnosed, with an estimated U.S. incidence of approximately 16,000 cases diagnosed annually (1). STS comprises a histopathologically heterogeneous group of mesenchymal tumors, consisting of approximately 50 subtypes (2), with challenging diagnosis and treatment pathways. Surgical excision remains the cornerstone of therapy for localized primary STS and is usually combined with neoadjuvant or adjuvant radiotherapy. The main goal of surgery is to resect an STS with a sufficient margin, excising the tumor and sufficient surrounding normal tissue. A tumor-negative margin is an important prognostic factor for local recurrence and disease-free survival $(3,4)$. A tumorpositive margin, which occurs in $14 \%-34 \%$ of cases, results in local recurrence rates of approximately 35\% (5). On histology, a tumorpositive margin is defined by tumor extension in the inked resection margin. However, microscopic tumor extension within $1 \mathrm{~mm}$ of the surgical margin may also be considered a tumor-positive margin, as both conditions are associated with increased risk of local recurrence. When a margin is tumor-positive, repeated resection of the surgical cavity has to be considered (5). However, repeated resection can be challenging because of complex anatomy in the surgical field as a result of postoperative fibrosis and the presence of neurovascular structures, which increase the risk of morbidity. Therefore, in many cases, proper clinical decision making during surgery is a clinical trade-off in which achieving a tumor-negative surgical margin and functional outcome must be weighed. It is currently difficult for the surgeon to assess margin status during surgery, emphasizing the need for an intraoperative real-time imaging technique. During STS surgery, a clinician should be able to visualize tumor cells in 
the rim of the resection margin, both in vivo and ex vivo. Fluorescence-guided surgery (FGS) is an optical imaging method that provides real-time tumor detection and can be used to assess margins. Several studies have investigated the potential merits of FGS in malignancies such as breast cancer, peritoneal metastasis, colon cancer, glioblastoma, and head and neck cancer (6-11). These studies revealed promising data on how detecting residual disease can potentially benefit intraoperative decision making. For STS, only preclinical studies and one proof-of-concept study on a single patient have been reported, using ABY-029 and focusing on biomarkers such as epidermal growth factor receptor for FGS $(12,13)$.

In STS, a patient-tailored surgical plan is designed on the basis of tumor location and histopathologic tumor classification, using immunohistochemistry, molecular genetics, and tumor grading (14). Because high-grade STS with extracompartmental invasive growth has a relatively decreased overall survival and event-free survival, one should strive for an adequately wide en bloc resection (15). In particular, myxofibrosarcoma is associated with tumor-positive margins because of its diffuse reticular growth in subcutaneous fat or muscle, whereas other types of STS, such as myxoid liposarcomas, tend to have a pushing invasive border making them easier to excise, with narrow but clear margins (16).

Vascular endothelial growth factor A (VEGF-A) is involved in angiogenesis and lymph angiogenesis and is overexpressed in many solid tumors (17). Overexpression of VEGF-A in STS has been reported (18-20). The therapeutic monoclonal antibody bevacizumab (Avastin; Genentech) binds to VEGF-A. By conjugating bevacizumab to the organic fluorophore IRDye800CW, a tumor-specific tracer (bevacizumab-800CW) could be developed $(9,21,22)$. In previous studies using this tracer, bevacizumab-800CW was shown to be safe for use in humans.

The aim of this FGS study was to determine the feasibility, safety, and optimal dose of bevacizumab-800CW for in vivo and ex vivo detection and margin assessment of STS, using a standardized fluorescence imaging workflow $(22,23)$.

\section{MATERIALS AND METHODS}

This phase 1 single-center feasibility study was performed at the University Medical Center of Groningen. The study was approved by the Institutional Review Board (approval 2017/302) and was conducted according to the principles of Helsinki (adapted version; Fortaleza, Brazil; 2013) and the laws and regulations of The Netherlands. The trial was registered at www.clinicaltrials.gov (NCT03913806). All patients provided written informed consent before participation in the study.

\section{Patients}

Patients aged older than $18 \mathrm{y}$ with histopathologically proven STS and appropriate imaging (CT/MRI), and scheduled for surgical excision, were included. All patients had a World Health Organization performance score of $0-2$. Patients with concurrent invasive malignancy were excluded. Other exclusion criteria were medical or psychiatric conditions compromising the patient's ability to give informed consent; pregnancy or lactation; a history of infusion reactions to bevacizumab; inadequately controlled hypertension; or a history of myocardial infarction, transient ischemic attack, cerebrovascular accidents, pulmonary embolism, uncontrolled chronic hepatic failure, or unstable angina pectoris 6 mo before inclusion. All patients eligible for surgery and their inclusion in the trial were discussed in the multidisciplinary sarcoma team meeting before surgery.

\section{Study Design}

The primary objective of the study was to determine the feasibility of bevacizumab-800CW in STS for in vivo and ex vivo tumor detection. Secondary objectives were to identify the optimal dose for visualization of STS tissue and to obtain information on safety aspects of the tracer in STS patients. A classic $3 \times 3$ dose-finding study design was used, consisting of 2 parts. In the dose-escalation phase (part 1), 3 dose cohorts were tested. Three subjects per cohort received intravenously administered flat doses of 10,25 , or $50 \mathrm{mg} 3 \mathrm{~d}$ before surgery. In the dose-expansion phase (part 2), the optimal dosing group (the initial subgroup of 3 patients) was expanded up to 10 subjects. The optimal dose group was based on in vivo and ex vivo tumor-to-background ratio (TBR). The study design was adapted after the conclusion of part 1 to exclude patients who underwent neoadjuvant treatment in part 2 .

\section{Safety Assessment}

Vital signs were measured before, directly after, and $1 \mathrm{~h}$ after tracer administration. Follow-up was performed up to $2 \mathrm{wk}$ after tracer administration to assess adverse events, which were scored according to the National Cancer Institute Common Terminology Criteria for Adverse Events, version 4.0.

\section{Bevacizumab-800CW Production}

Bevacizumab-800CW was produced in the good-manufacturingpractice facility of the Department of Clinical Pharmacy and Pharmacology at the University Medical Center of Groningen, as reported previously (21).

\section{Intraoperative Fluorescence Imaging}

The tumor was fluorescently imaged during surgery using Explorer Air (SurgVision BV), as described earlier (22). Immediately after tumor excision (i.e., $<5 \mathrm{~min}$ ), fluorescence imaging of all resection planes of the surgical specimen was performed with Explorer Air coupled to a closed-field imaging box (The Vault; the SurgVision Explorer Air). Within $1 \mathrm{~h}$ after excision, imaging was also performed with the Pearl Trilogy imaging system (LI-COR BioSciences). The acquisition settings for Explorer Air and Pearl Trilogy were as described previously by our group for both in vivo and ex vivo imaging (22). No surgical decisions were based on fluorescence imaging.

\section{Fluorescence-Guided Pathology}

The fresh surgical specimen was inked and serially sliced following standard protocols for pathologic specimen handling. Fluorescence images of both sides of the tissue slices were obtained using Pearl Trilogy. Next, tissue slices were formalin-fixed for 1-4 d and imaged with Pearl Trilogy before embedding (Fig. 1). A pathologist, masked to fluorescence, selected clinically relevant regions for further formalin fixation and paraffin embedding. On the basis of fluorescence imaging, additional regions of interest were selected for additional formalin fixation and paraffin embedding. A standard fluorescence-guided workflow was used to cross-correlate the fluorescent tissue slices to the final histopathology (based on H\&E staining) (22). Hematoxylin and eosin (H\&E)-stained slides were reviewed by a pathologist masked to the results of fluorescence imaging. The complete workflow of the study is depicted in Figure 1.

\section{Fluorescence Quantification}

The in vivo TBR was calculated as the mean fluorescence intensity (MFI) of the tumor divided by the MFI of the background. The tumor was delineated on macroscopic visualization. The background MFI was calculated on all nontumor tissue surrounding the tumor in the surgical field. MFI (arbitrary units) was calculated on Explorer Air images (exposure time, 25 or $50 \mathrm{~ms}$; gain, 10-100). For the whole-specimen imaging immediately after excision, the maximum fluorescence intensities 


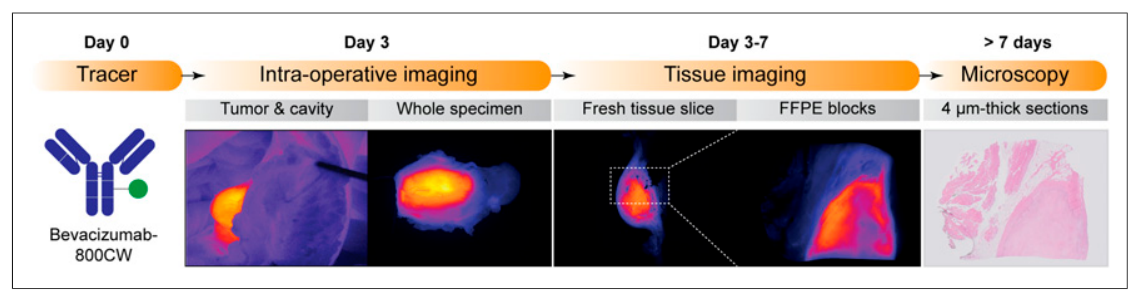

FIGURE 1. Standardized fluorescence imaging workflow. Patients received bevacizumab$800 \mathrm{CW}$ intravenously $3 \mathrm{~d}$ before surgery. During surgery, tumor was fluorescently imaged in vivo using Explorer Air. Directly after excision, whole surgical specimen was imaged using Explorer Air and Pearl Trilogy. After serial slicing by pathologist, fluorescence images of all steps of histopathologic processing were sliced serially and cross-correlated with standard-of-care H\&E slides. FFPE $=$ formalin-fixed, paraffin-embedded.

were calculated on the whole resection margin to correlate signal intensity to margin depth.

Ex vivo TBRs were calculated on fresh serially sliced tissues. The tumor and the surrounding nontumor tissue were precisely delineated on standard H\&E histopathologic slides by a pathologist masked to fluorescence. An overlay with fluorescent tissue slices was based on anatomic landmarks. Afterward, the ex vivo TBR was calculated as the MFI of the tumor divided by the MFI of the background. The background MFI was calculated on all nontumor tissue for every tissue slice.

\section{Statistics}

Descriptive statistics were reported as mean with SD in cases of normal distribution, whereas median with interquartile range was used in cases of skewed distribution. Fluorescence signals in tumor and normal tissue were compared using the Mann-Whitney test. A $P$ value of less than 0.05 was considered statistically significant. For descriptive statistics, SPSS (version 23.0; SPSS Inc.) was used.

\section{RESULTS}

Between April and December 2019, 15 patients with 7 different histopathologic subtypes of STS, including 7 myxofibrosarcomas, were enrolled in this dose-escalation trial. Patient, safety, and tumor characteristics are depicted in Table 1 and Supplemental Table 1 (supplemental materials are available at http://jnm.snmjournals.org). Bevacizumab-800CW was administered $3 \mathrm{~d}$ before surgery to all patients. No tracer-related serious adverse events were observed (Supplemental Table 1).

\section{Dose Escalation Phase}

Eight patients were included in the dose-escalation phase $(10 \mathrm{mg}$, $n=3 ; 25 \mathrm{mg}, n=3$; and $50 \mathrm{mg}, n=2$ ). All tumors were adequately visualized in vivo regardless of dose (TBR: $2.0 \pm 0.58$ for $10 \mathrm{mg} ; 2.5 \pm$ 0.32 for $25 \mathrm{mg}$; and $2.0 \pm 0.10$ for $50 \mathrm{mg}$ ) (Fig. 2). After excision, tumor tissue was adequately visualized on the excised specimen (TBR: $2.0 \pm 1.1$ for $10 \mathrm{mg}$ and $2.5 \pm 0.31$ for $25 \mathrm{mg}$ ). No reliable ex vivo TBR could be calculated for the $50 \mathrm{mg}$ group because of lack of nontumor background tissue. Since no increase in in vivo TBR was obtained for the $50 \mathrm{mg}$ cohort after 2 patients, only 2 patients were enrolled in this cohort. Because there were no statistical differences between the in vivo $10 \mathrm{mg}$ and $25 \mathrm{mg}$ cohorts, $(P=0.18)$, the $10 \mathrm{mg}$ cohort was considered to be the optimal dosing group and was expanded by 7 additional patients.

\section{Ex Vivo Fluorescence and Quantification on Tissue Slices}

To quantify fluorescence, fresh tissue slices were used. All 7 histopathologic STS subtypes could be visualized ex vivo, displaying a sharp, clearly delineated fluorescent signal in all tissue slices containing tumor (Fig. 3). Significantly higher fluorescence signals were seen in tumor tissue than in nontumor tissue in the $10 \mathrm{mg}$ group (Fig. 3, $n=22$, $P<0.001$, median MFI of 0.013 for tumor tissue and 0.004 for nontumor tissue). The median ex vivo TBRs of the 10- and $25 \mathrm{mg}$ dose cohorts were $2.67 \pm 1.6(n=9$; range, 1.5-6.5) and 4.6 $\pm 0.31(n=2$; range, 4.5-4.7), respectively. In the STS series, all myxofibrosarcomas $(n=7)$ could be visualized using fluorescence during ex vivo imaging (Supplemental Fig. 1). Because of the imaging resolution of the Pearl Trilogy, the border zone with strands of diffusely infiltrating tumor cells could not be individually identified with visual fluorescence inspection. In this study, we found it easier to identify tumor areas that were more solid and cellular.

In the tissue slices, a false-positive fluorescent signal could be observed in areas with a high macrophage content. We suspect that

TABLE 1

Clinical and Pathologic Characteristics

\begin{tabular}{|c|c|}
\hline Characteristic & Data \\
\hline Age at surgery $(\mathrm{y})$ & $66(34-84)$ \\
\hline Sex: male & $8(53)$ \\
\hline Weight (kg) & $82(64-103)$ \\
\hline Height (cm) & $174(161-189)$ \\
\hline \multicolumn{2}{|l|}{ Dosing groups } \\
\hline $10 \mathrm{mg}$ & $10(67)$ \\
\hline $25 \mathrm{mg}$ & $3(20)$ \\
\hline $50 \mathrm{mg}$ & $2(13)$ \\
\hline Tracer-related adverse events & $0(0)$ \\
\hline \multicolumn{2}{|l|}{ Type of primary tumor } \\
\hline Myxofibrosarcoma & $7(47)$ \\
\hline High-grade & $6(86)$ \\
\hline Mixed type & $1(14)$ \\
\hline Liposarcoma & $3(20)$ \\
\hline Synovial sarcoma & $2(13)$ \\
\hline Leiomyosarcoma & $1(7)$ \\
\hline Angiosarcoma & $1(7)$ \\
\hline Undifferentiated pleomorphic sarcoma & $1(7)$ \\
\hline Tumor diameter (mm) & $112(18-320)$ \\
\hline \multicolumn{2}{|l|}{ Location of tumor } \\
\hline Arms & $4(27)$ \\
\hline Legs & $4(27)$ \\
\hline Intraabdominal & $4(27)$ \\
\hline Hip or flank & $2(13)$ \\
\hline Breast & $1(7)$ \\
\hline
\end{tabular}

Qualitative data are numbers and percentages; continuous data are means and ranges (total patients $=15$ ). 


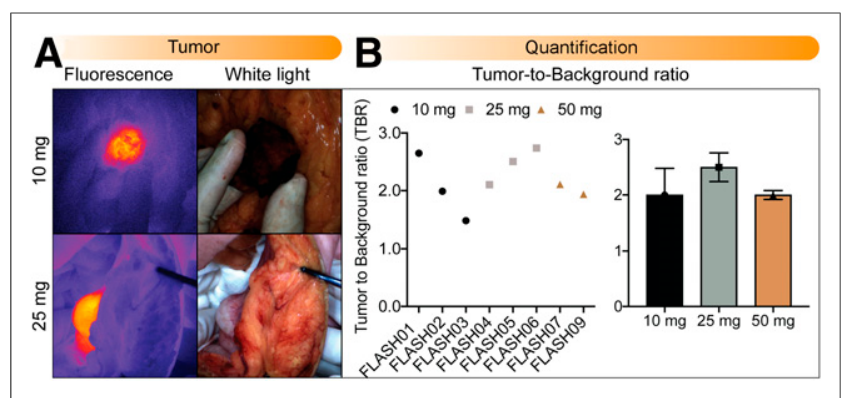

FIGURE 2. In vivo visualization of STS. (A) Intraoperative imaging was performed with Explorer Air. Fluorescence and white-light images were obtained in vivo using $10 \mathrm{mg}$ of bevacizumab-800CW for intraabdominal leiomyosarcoma or $25 \mathrm{mg}$ of bevacizumab-800CW for synovial sarcoma. (B). Median in vivo TBRs for all patients in dose escalation phase $(n=8)$ and per dosing group were calculated.

this finding was due to an inflammatory response after tumor necrosis induced by neoadjuvant radiotherapy (Supplemental Figs. $1 \mathrm{~A}-1 \mathrm{C}$ and $1 \mathrm{M}-1 \mathrm{O})$. For this reason, patients who received neoadjuvant radiotherapy were not eligible to participate during the dose-expansion phase, to minimize false-positive nontumor tissue signal.

\section{Fluorescence Margin Assessment}

After histopathologic assessment, 7 patients were diagnosed with a tumor-positive surgical margin (47\%), including 3 high-grade myxofibrosarcomas, 2 high-grade undifferentiated sarcomas, and 1 synovial sarcoma. In 1 retroperitoneal well-differentiated and focally dedifferentiated liposarcoma, the margin was considered positive because of the extent of the disease and the limitations in surgical

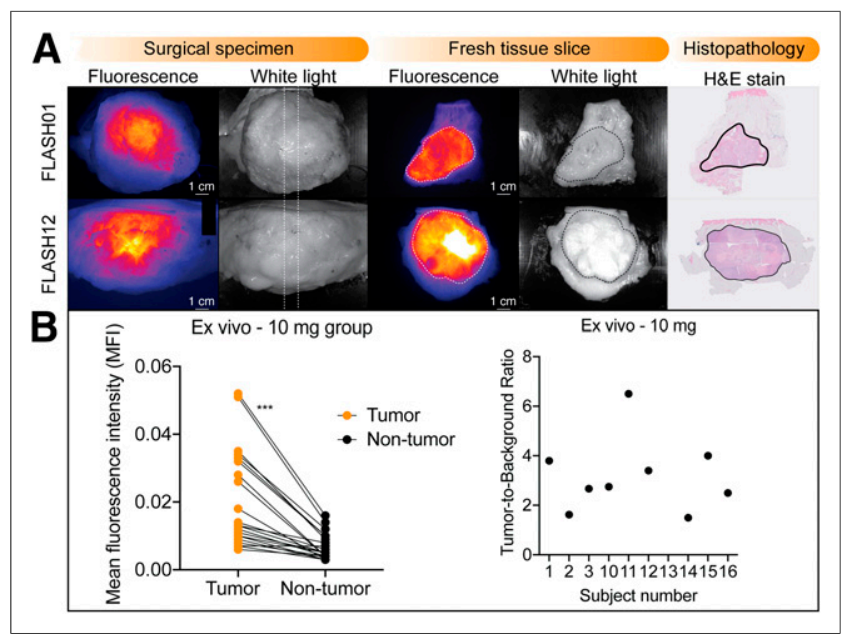

FIGURE 3. Ex vivo visualization of STS. (A) Leiomyosarcoma and myxofibrosarcoma are shown, with whole-specimen image of close surgical margins on basal $(0.6 \mathrm{~cm})$ and caudal $(0.6 \mathrm{~cm})$ resection margins. A sharply delineated tumor-specific fuorescence signal on the tissue slices is observed. The tumor is delineated with a dashed line and close surgical margins on the basal and caudal resection margin. Tumors are delineated with solid line on standard H\&E staining. (B) Subanalysis in $10 \mathrm{mg}$ group showed significantly higher MFI in all tumor tissue than in nontumor tissue $(n=22$, $P<0.001)(\mathrm{k})$, and all ex vivo TBRs are presented for all patients in $10 \mathrm{mg}$ group $(n=8)$. No data are available for FLASH03 (false-positive signal) or FLASH13 (angiosarcoma, no reliable calculation possible). excision, but this case was not included in our fluorescence analysis. All tumor-positive surgical margins were detected using fluorescence imaging of the resected specimen (7/7). Three patients had a histologically proven tumor-negative surgical margin (20\%), and fluorescence predicted 1 of the 3 correctly (33\%). One margin was false-positive because of neoadjuvant treatment, whereas the other margin was false-positive because of a highly fluorescent cyst. Five patients had a histologically proven close surgical margin (33\%) ranging from 0.6 to $1.4 \mathrm{~mm}$, one of which was false-positive for fluorescence because of neoadjuvant treatment and was therefore excluded from our analysis $(n=4)$. Quantification of maximum fluorescence intensities on whole-specimen imaging with a close surgical margin $(0.6-1.4 \mathrm{~mm})$ showed a nonsignificant trend in maximum fluorescence intensities on the resection margins with the closest surgical margin (Fig. 3). For all patients ( $n=4,13$ resection margins evaluated) with a close surgical margin (range, 0.6-1.4 mm), the surgical side with the closest surgical margin had the highest maximum fluorescence intensity, suggesting a relation between maximum fluorescence intensity and margin depth (Fig. 4).

\section{DISCUSSION}

In this pilot study, we showed that FGS using bevacizumab$800 \mathrm{CW}$ is feasible and safe in a variety of histologic subtypes of STS using both in vivo and ex vivo fluorescence imaging. The $10 \mathrm{mg}$ dose of bevacizumab-800CW proved to be safe and sufficient for adequate in vivo and ex vivo imaging, whereas a dose of 25 or $50 \mathrm{mg}$ did not significantly improve fluorescence visualization. Moreover, we showed that FGS has great potential value for tumor detection and margin assessment during STS surgery. This potential was evident after studying depth assessment for close surgical margins using ex vivo fluorescence-guided imaging of specimens immediately after excision, as shown in previous FGS studies $(22,24)$. We believe that this technique has the potential to optimize surgical quality and decrease the number of tumor-positive surgical margins.

Tumor histologic type and grade, as well as the anatomic location of STS, are important determinants of local recurrence. Ideally, FGS should reflect tumor infiltration patterns (diffuse vs. pushing infiltrative borders) during surgery. Our data on fluorescence visualization of myxofibrosarcomas confirm that this tumor is accompanied by frequent positive surgical margins due to diffuse, reticular invasive growth in surrounding soft tissue. Despite the fact that the solid cellular tumor areas could be easily identified, future studies should determine whether FGS can enhance intraoperative margin assessment in diffusely infiltrative STS.

In our previous studies on breast cancer, we confirmed the colocalization of bevacizumab-800CW with VEGF-A IHC. In $90 \%$ of all patients, there was adjacent or complete overlap of bevacizumab-800CW and VEGF-A immunohistochemistry staining, which also could be observed in immunohistochemistry analysis of STS tissue (Supplemental Fig. 2) (10). As VEGF-A staining is heterogeneous in different STS subtypes and would be reliable only if thoroughly analyzing the whole surgical specimen, we did not include a full immunohistochemistry analysis in this study. Nevertheless, VEGF-A expression has recently been observed as a suitable marker for FGS in $93 \%$ of myxofibrosarcomas, and VEGF-A expression significantly correlated with clinical variables such as high histologic grade and distant metastasis $(25,26)$. Our imaging results, showing specific fluorescence activation in areas containing soft-tissue tumor as assessed by H\&E staining, 


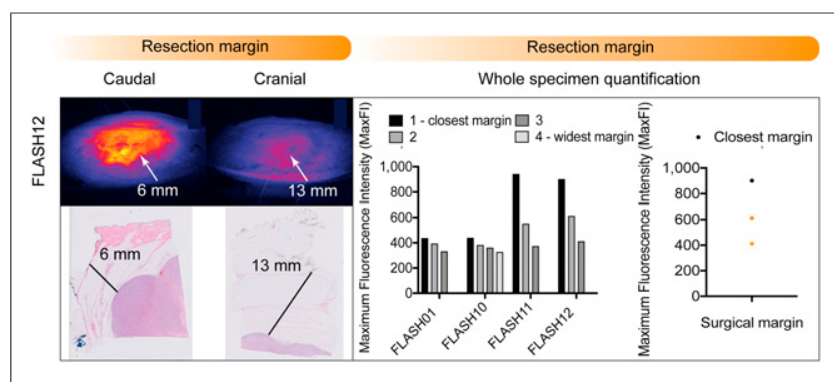

FIGURE 4. Ex vivo whole-specimen imaging directly after excision. Immediately after surgical excision ( $<5 \mathrm{~min})$, standardized fluorescence images were obtained of all resection planes of surgical specimen. In this myxofibrosarcoma, caudal resection margin showed high maximum fluorescence intensity (901), which correlated with close surgical margin of $6 \mathrm{~mm}$, whereas cranial resection margin showed lower maximum fluorescence intensity (612), correlating with deeper resection margin of $13 \mathrm{~mm}$. Maximum-fluorescence-intensity signals were calculated for all close resection margins in 4 patients. Margin of 1 is closest surgical margin as described by standard histopathology, and margin of 4 is widest. Per-patient analysis for patient FLASH12 is presented.

confirm these preclinical results and are in accordance with clinical data reported earlier. Tumor necrosis, frequently observed in highgrade STS, may be induced by neoadjuvant radiotherapy. In general, tumor necrosis is a significant predictor of relapse-free survival and overall survival in STS patients (27). Bevacizumab-800CW is not adequately visualized in necrotic areas, as no adequate penetration and specific binding could be obtained. We observed falsepositive fluorescent signals in areas with high macrophage content and reactive fibrosis related to the inflammatory response after neoadjuvant radiotherapy. Therefore, FGS with bevacizumab-800CW for real-time margin assessment seems to be most suitable during primary surgery with radiotherapy in an adjuvant setting. In a phase 2 study, a cohort with a significant number of neoadjuvantly treated patients should be included to compare the results of FGS in patients with STS undergoing neoadjuvant or adjuvant treatment.

As previously described in head and neck specimens, quantification of all resection planes of the whole specimen directly after surgical excision using a fluorescence signal surface map may identify margin depth with fluorescence intensity peaks. The margin segment with the highest fluorescence intensity was termed the sentinel margin - the location where the closest margin is mostly likely to be located (28). Although we could correlate the maximum fluorescence intensity to a close surgical margin, our study was not powered to determine the invasion depth on a microscopic level. Currently, it is challenging to determine sentinel margins in sarcoma surgery, as fluorescence imaging of, especially, the deep resection margins is not feasible using the currently available large intraoperative imaging devices. Sarcomas are usually in tight anatomic spaces requiring challenging positioning of intraoperative devices. Furthermore, the variation in imaging acquisition settings and the absence of standardization may result in a lack of quality control. Ex vivo fluorescence imaging with so-called closed-field cameras enables better control of image acquisition parameters and removes interference from ambient light. Our ex vivo analysis can be performed within 5-10 min after surgical excision, as ex vivo imaging enables a standardized and reproducible image analysis platform to guide surgical decision making. Standardizing fluorescence imaging could minimize signal inhomogeneity that can lead to erroneous tumor delineation. In a future scenario, on-site examination in the surgical theater by a pathologist equipped with a fluorescence imaging system may guide the surgeon in adapting surgical treatment decisions. On the basis of intraoperative imaging findings and the outcome of fluorescence-guided pathology, this ability may potentially prevent both under- and overtreatment and diminish surgical reinterventions at a later stage.

\section{CONCLUSION}

This study is a first proof of the concept that FGS using a flat dose of $10 \mathrm{mg}$ of bevacizumab- $800 \mathrm{CW}$ is feasible in a variety of STS subtypes. Moreover, the potential value of fluorescence imaging during ex vivo pathology assessment was evident. FGS appears to be most valuable in patients undergoing primary surgery without neoadjuvant treatment. A subsequent phase 2 trial will be performed in our institute to determine the sensitivity and specificity of FGS in patients treated with primary surgery with curative intent.

\section{DISCLOSURE}

The research leading to the results was supported by an unrestricted research grant from SurgVision BV. Gooitzen van Dam is a consultant for OncoNano Medicine Inc. and is the CEO, the founder, and a shareholder of the AxelaRx/TRACER BV group. No other potential conflict of interest relevant to this article was reported.

\section{ACKNOWLEDGMENTS}

We thank the pathology and microbiology departments, in particular Maaike Barentsen, Eric Bleuel, Lilo Janssens, Marina López-Álvarez, and Gert Jan Meersma, for excellent technical assistance.

\section{KEY POINTS}

QUESTION: The incidence of tumor-positive surgical margins in STS is high, and in this clinical research we investigated whether FGS using a targeted imaging approach with bevacizumab$800 \mathrm{CW}$ for in vivo and ex vivo tumor detection has the potential to decrease the number of tumor-positive surgical margins.

PERTINENT FINDINGS: All STSs could be visualized during in vivo and ex vivo imaging. A flat dose of $10 \mathrm{mg}$ of bevacizumab$800 \mathrm{CW}$ was optimal, and all tumor-positive margins could be observed in real time by back-table fluorescence imaging immediately after surgical resection.

IMPLICATIONS FOR PATIENT CARE: The potential for improved tumor detection might optimize surgical quality and decrease the number of tumor-positive surgical margins.

\section{REFERENCES}

1. Siegel RL, Miller KD, Jemal A. Cancer statistics, 2019. CA Cancer J Clin. 2019;69:7-34.

2. Dickinson IC, Whitwell DJ, Battistuta D, et al. Surgical margin and its influence on survival in soft tissue sarcoma. ANZ J Surg. 2006;76:104-109.

3. Gronchi A, Lo Vullo S, Colombo C, et al. Extremity soft tissue sarcoma in a series of patients treated at a single institution. Ann Surg. 2010;251:506-511.

4. Kainhofer V, Smolle MA, Szkandera J, et al. The width of resection margins influences local recurrence in soft tissue sarcoma patients. Eur J Surg Oncol. 2016;42:899-906.

5. Gundle KR, Kafchinski L, Gupta S, et al. Analysis of margin classification systems for assessing the risk of local recurrence after soft tissue sarcoma resection. J Clin Oncol. 2018;36:704-709. 
6. van Dam GM, Themelis G, Crane LMA, et al. Intraoperative tumor-specific fluorescence imaging in ovarian cancer by folate receptor- $\alpha$ targeting: first inhuman results. Nat Med. 2011;17:1315-1319.

7. Rosenthal EL, Warram JM, de Boer E, et al. Safety and tumor specificity of cetuximab-IRDye 800 for surgical navigation in head and neck cancer. Clin Cancer Res. 2015;21:3658-3666.

8. Whitley MJ, Cardona DM, Lazarides AL, et al. A mouse-human phase 1 coclinical trial of a protease-activated fluorescent probe for imaging cancer. Sci Transl Med. 2016;8:320ra4.

9. Harlaar NJ, Koller M, de Jongh SJ, et al. Molecular fluorescence-guided surgery of peritoneal carcinomatosis of colorectal origin: a single-centre feasibility study. Lancet Gastroenterol Hepatol. 2016;1:283-290.

10. Lamberts LE, Koch M, de Jong JS, et al. Tumor-specific uptake of fluorescent bevacizumab-IRDye $800 \mathrm{CW}$ microdosing in patients with primary breast cancer: a phase I feasibility study. Clin Cancer Res. 2017;23:2730-2741.

11. Zeh R, Sheikh S, Xia L, et al. The second window ICG technique demonstrates a broad plateau period for near infrared fluorescence tumor contrast in glioblastoma. Bogyo M, ed. PLoS One. 2017;12:e0182034.

12. Samkoe KS, Sardar HS, Bates BD, et al. Preclinical imaging of epidermal growth factor receptor with ABY-029 in soft-tissue sarcoma for fluorescence-guided surgery and tumor detection. J Surg Oncol. 2019;119:1077-1086.

13. Samkoe KS, Sardar HS, Gunn J, et al. Measuring microdose ABY-029 fluorescence signal in a primary human soft-tissue sarcoma resection. Proc SPIE-the Int Soc Opt Eng. 2019;10862.

14. Canter RJ. Surgical approach for soft tissue sarcoma: standard of care and future approaches. Curr Opin Oncol. 2015;27:343-348.

15. Tsukushi S, Nishida Y, Urakawa H, Kozawa E, Ishiguro N. Prognostic significance of histological invasion in high grade soft tissue sarcomas. SpringerPlus. 2014;3:544.

16. Baxter KJ, Govsyeyev N, Namm JP, Gonzalez RJ, Roggin KK, Cardona K. Is multimodality therapy necessary for the management of pure myxoid liposarcomas? A multi-institutional series of pure myxoid liposarcomas of the extremities and torso. J Surg Oncol. 2015;111:146-151.

17. Hicklin DJ, Ellis LM. Role of the vascular endothelial growth factor pathway in tumor growth and angiogenesis. J Clin Oncol. 2005;23:1011-1027.
18. Chao C, Al-Saleem T, Brooks JJ, Rogatko A, Kraybill WG, Eisenberg B. Vascular endothelial growth factor and soft tissue sarcomas: tumor expression correlates with grade. Ann Surg Oncol. 2001;8:260-267.

19. Kilvaer TK, Valkov A, Sorbye S, et al. Profiling of VEGFs and VEGFRs as prognostic factors in soft tissue sarcoma: VEGFR-3 is an independent predictor of poor prognosis. PLoS One. 2010;5:e15368.

20. Wanebo HJ, Argiris A, Bergsland E, Agarwala S, Rugo H. Targeting growth factors and angiogenesis; using small molecules in malignancy. Cancer Metastasis Rev. 2006;25:279-292.

21. Ter Weele EJ, Terwisscha van Scheltinga AGT, Linssen MD, et al. Development, preclinical safety, formulation, and stability of clinical grade bevacizumab$800 \mathrm{CW}$, a new near infrared fluorescent imaging agent for first in human use. Eur J Pharm Biopharm. 2016;104:226-234.

22. Koller M, Qiu S-Q, Linssen MD, et al. Implementation and benchmarking of a novel analytical framework to clinically evaluate tumor-specific fluorescent tracers. Nat Commun. 2018;9:3739.

23. Tummers WS, Warram JM, van den Berg NS, et al. Recommendations for reporting on emerging optical imaging agents to promote clinical approval. Theranostics. 2018;8:5336-5347.

24. Rosenthal EL, Warram JM, De Boer E, et al. Successful translation of fluorescence navigation during oncologic surgery: a consensus report. $\mathrm{J}$ Nucl Med. 2016;57:144-150.

25. Diao Y, Zhang P, Dai R, Xu J, Feng H. H3K27me3 and VEGF is associated with poor prognosis in patients with synovial sarcoma. Pathol Res Pract. 2018;214:974-977.

26. de Gooyer JM, Versleijen-Jonkers YMH, Hillebrandt-Roeffen MHS, et al. Immunohistochemical selection of biomarkers for tumor-targeted image-guided surgery of myxofibrosarcoma. Sci Rep. 2020;10:2915.

27. Salah S, Lewin J, Amir E, Abdul Razak A. Tumor necrosis and clinical outcomes following neoadjuvant therapy in soft tissue sarcoma: a systematic review and meta-analysis. Cancer Treat Rev. 2018;69:1-10.

28. van Keulen S, Nishio N, Birkeland A, et al. The sentinel margin: intraoperative ex vivo specimen mapping using relative fluorescence intensity. Clin Cancer Res. 2019;25:4656-4662. 